\title{
In vivo biocompatibility of new nano-calcium- deficient hydroxyapatite/poly-amino acid complex biomaterials
}

This article was published in the following Dove Press journal:

International Journal of Nanomedicine

6 October 2015

Number of times this article has been viewed

\author{
Zhenyu Dai ${ }^{1,2, *}$ \\ Yue $\mathrm{Li}^{3, *}$ \\ Weizhong $\mathrm{Lu}^{2}, *$ \\ Dianming Jiang ${ }^{4}$ \\ Hong $\mathrm{Li}^{\prime}$ \\ Yonggang Yan' \\ Guoyu Lv' \\ Aiping Yang'
}

'College of Physical Science and Technology, Sichuan University,

Chengdu, ${ }^{2}$ Department of

Orthopedics, Chongqing Hospital

of Traditional Chinese Medicine,

${ }^{3}$ Department of Clinical Laboratory,

the Second Affiliated Hospital,

${ }^{4}$ Department of Orthopedics, the

First Affiliated Hospital, Chongqing

Medical University, Chongqing,

People's Republic of China

*These authors contributed equally to this work
Correspondence: Hong Li; Yonggang Yan College of Physical Science and Technology, Sichuan University, Chengdu 610064, People's Republic of China

Tel +8602885 I 27592

Fax +86028 85I87573

Email56443848I@qq.com;

yan_yonggang@vip.163.com
Objective: To evaluate the compatibility of novel nano-calcium-deficient hydroxyapatite/ poly-amino acid (n-CDHA/PAA) complex biomaterials with muscle and bone tissue in an in vivo model.

Methods: Thirty-two New Zealand white rabbits were used in this study. Biomaterials were surgically implanted into each rabbit in the back erector spinae and in tibia with induced defect. Polyethylene was implanted into rabbits in the control group and n-CDHA/PAA into those of the experimental group. Animals were examined at four different points in time: 2 weeks, 4 weeks, 12 weeks, and 24 weeks after surgery. They were euthanized after embolization. Back erector spinae muscles with the surgical implants were examined after hematoxylin and eosin (HE) staining at these points in time. Tibia bones with the surgical implants were examined by $\mathrm{X}$-ray and scanning electron microscopy (SEM) at these points in time to evaluate the interface of the bone with the implanted biomaterials. Bone tissues were sectioned and subjected to HE, Masson, and toluidine blue staining.

Results: HE staining of back erector spinae muscles at 4 weeks, 12 weeks, and 24 weeks after implantation of either n-CDHA/PAA or polyethylene showed disappearance of inflammation and normal arrangement in the peripheral tissue of implant biomaterials; no abnormal staining was observed. At 2 weeks after implantation, X-ray imaging of bone tissue samples in both experimental and control groups showed that the peripheral tissues of the implanted biomaterials were continuous and lacked bone osteolysis, absorption, necrosis, or osteomyelitis. The connection between implanted biomaterials and bone tissue was tight. The results of HE, Masson, toluidine blue staining and SEM confirmed that the implanted biomaterials were closely connected to the bone defect and that no rejection had taken place. The n-CDHA/PAA biomaterials induced differentiation of a large number of chondrocytes. New bone trabecula began to form at 4 weeks after implanting n-CDHA/PAA biomaterials, and lamellar bone gradually formed at 12 weeks and 24 weeks after implantation. Routine blood and kidney function tests showed no significant changes at 2 weeks and 24 weeks after implantation of both biomaterials.

Conclusion: n-CDHA/PAA composites showed good compatibility in in vivo model. In this study, n-CDHA/PAA were found to be safe, nontoxic, and biologically active in bone repair.

Keywords: in vivo implantation, histological evaluation, n-CDHA/PAA, bioactive composite

\section{Introduction}

Trauma- and tumor-induced bone defects are problems that can emerge after surgery. ${ }^{1}$ Although bone autograft and allograft implants have been used clinically to address bone defects, multiple bone autografts can burden patients with numerous adverse effects and so cause considerable suffering; in addition, implantation of allografts 
may induce rejection and other problems. ${ }^{2-4}$ The development of an ideal artificial restoration material has become an important topic in bone tissue engineering. Nano-calciumdeficient hydroxyapatite (n-CDHA) and poly-amino acid (PAA) undergo in situ polymerization to form n-CDHA/ PAA composites. These composites were recently produced in the current laboratory for the first time and may serve as bone repair biomaterials..$^{5}$ The copolymers of these amino acids were based on 6-aminocaproic acid as a main chain and $\alpha$-amino acids of human body as copolymerized units. The 6-aminocaproic acid gives the copolymer good mechanical properties and process abilities to polymers. Copolymerization of other natural amino acid monomers can produce polymers with different physical and chemical properties (eg, affinity, hydrophobic properties, degradation rates, degradation product, and $\mathrm{pH}) .{ }^{6} \mathrm{n}-\mathrm{CDHA}$ bases on the particle structure (particle diameter of 80-100 nm) cause the particle to disperse in PAA matrix biomaterial uniformly, allowing two types of chemical bonds on the interface of composites between n-CDHA and PAA: - $\mathrm{COO}^{-}$ions of PAA replace the anions $\left(\mathrm{OH}^{-}\right.$and $\left.\mathrm{Po}_{4}^{3-}\right)$ of n-CDHA and form a strong chemical bond with $\mathrm{Ca}^{2+}$ ions; O-H of n-CDHA forms hydrogen bonds with the amide bond in the polymer chain. These two types of combinations allow very good transmission of force and dispersion of stress, improving the mechanical properties of the biomaterials. ${ }^{6}$ A previous study showed that n-CDHA/ PAA composites had good mechanical properties and cell compatibility. ${ }^{5}$ To further explore the possibility of applying $\mathrm{n}-\mathrm{CDHA} / \mathrm{PAA}$ in the repair of human bone tissue and their biocompatibility in in vivo tissues, this study implanted n-CDHA/PAA composites into erector spinae and tibia bone of experimental animals.

\section{Materials and methods \\ Materials}

$\phi 6 \times 2 \mathrm{~mm}$ polyethylene (PE) and n-CDHA/PAA composites were provided by Sichuan International Nano Co., Ltd. (Sichuan, People's Republic of China). n-CDHA/PAA composites were legally prepared by in situ polymerization. ${ }^{7,8}$ n-CDHA mass fraction of n-CDHA/PAA was $30 \mathrm{wt} \%$. PAA contains six kinds of amino acids: 6-aminocaproic acid, glycine, L-alanine, L-phenylalanine, L-proline, and L-lysine. All engineered implants were sterilized using ethylene oxide.

\section{Experimental animals and grouping}

A total of 32 New Zealand white rabbits were provided by Laboratory Animal Center, Chongqing Medical University (Chongqing, People's Republic of China), of both sexes and body weight ranging from $2.0 \mathrm{~kg}$ to $2.5 \mathrm{~kg}$. For all animals, breeding conditions were kept the same before and during the study. Animals were divided into n-CDHA/PAA experimental group and $\mathrm{PE}$ control group. All animals received humane care in compliance with the Public Health Service Policy on Humane Care and Use of Laboratory Animals. Ethical approval was obtained from the Animal Care and Ethics Committee of Chongqing Medical University of China.

\section{Methods}

All 32 animals were anesthetized with 3\% sodium pentobarbital $(1 \mathrm{~mL} / \mathrm{kg})$ intravenously via the ear. Each animal was fixed in a supine position. The skin was prepared in the conventional manner, including disinfection and placement of surgical drapes. A longitudinal skin incision $\sim 2 \mathrm{~cm}$ in length was made along the lateral tibia of the upper two-thirds of rabbit calf, followed by separation of the subcutaneous tissue, fascia, and muscle to expose the proximal tibia. Upper and lower holes were drilled outward from the lateral side of tibia to the contralateral cortex, with $\sim 10 \mathrm{~mm}$ spacing and $2 \mathrm{~mm}$ diameter. n-CDHA/PAA and PE biomaterials were implanted into animals in the experimental and the control groups, followed by suturing and topical skin closure, disinfection, and application of erythromycin ointment to the incisions. Each animal that received an intramuscular implant was fixed in a prone position, followed by conventional skin preparation, disinfection, and placement of surgical drapes. Longitudinal incision along the back of the skin was performed, followed by separation of the subcutaneous tissue and superficial fascia to expose the erector spinae muscle on both sides. This muscle was blunt dissection. In the experimental group, two pieces of n-CDHA/PAA biomaterials were longitudinally implanted in each side of the erector muscle (with spacing of $2 \mathrm{~cm}$ ). A total of four pieces of n-CDHA/PAA biomaterial were implanted into each animal. In the control group, PE biomaterials were implanted into each animal as in the experimental group. All animals were sutured to close the skin. This was followed by disinfection and application of erythromycin ointment to the incisions. Each animal received intramuscular injection of penicillin (200,000 units daily) in the first 3 days after the surgery.

\section{Extraction and observation index of implant biomaterials}

Postoperative conditions, general food intake, and incision sites of each animal were examined at 2 weeks, 4 weeks, 12 weeks, and 24 weeks, followed by embolization to euthanize the animals in both groups (four animals per point in time; 16 animals per group). Approximately $5-10 \mathrm{~mm}$ of muscle 
surrounding the intramuscular implanted biomaterials was dissected. General conditions of the peripheral muscle of the implant biomaterials were observed with the naked eye. Three samples were randomly selected from each group at each point in time for the hematoxylin and eosin (HE) staining. Areas of tibia bones with implanted biomaterials underwent frontal and lateral X-ray imaging at the specified points in time to show the interface of the implant biomaterials with the animal's own tissue. Animals in both groups were euthanized after embolism at the specified points in time. Approximately $10 \mathrm{~mm}$ width of the bone tissue surrounding the implanted biomaterials were extracted along the original incision. General conditions of the tissue surrounding the implanted biomaterials were observed with the naked eye. Two samples were randomly selected from both groups at each point in time, and the soft tissue was carefully removed from the surface of implanted biomaterials. Samples were then fixed in $2 \%$ glutaraldehyde for scanning electron microscopy (SEM) of the implant-bone interface. Three samples were randomly selected from both groups at each point in time and fixed in formalin buffer. This was followed by hard-tissue sectioning and $\mathrm{HE}$, Masson, and toluidine blue staining to assess inflammation, foreign object rejection, immune response, and osteogenesis in the bone tissue around the implanted biomaterials. Three animals from each group were examined 2 weeks before and after the surgery and 24 weeks after the surgery. Blood was extracted for routine testing and analysis of biochemical markers for study of the impacts of implant biomaterials on animal blood, liver, and kidney functions.

\section{Statistical analysis}

SPSS16.0 software (SPSS Inc., Chicago, IL, USA) were used for statistical analysis. Results are presented as mean \pm standard deviation $(\bar{x} \pm s)$. Measurement data were analyzed by $t$-test. $P<0.05$ was considered indicative of statistically significant differences.

\section{Results}

\section{General condition of in vivo implantation}

The general condition of all animals was good after the surgery and showed no particular abnormalities, incision infection, or dehiscence of incision. Intramuscular embedded regions were examined at different points in time after n-CDHA/PAA implantation. The muscles had engulfed around the biomaterials, and no significant edema, infection, or necrosis had occurred in the peripheral muscles (Figure 1A and C); similar results were also observed in the control group (Figure 1B and D). The tibia bone area with implant biomaterials was collected at postoperative 2 weeks and showed that the peripheral bones of both implant biomaterials were closely connected. No significant necrosis, osteolysis, or bleaching of materials was observed in the bone tissues. In addition, no significant inflammatory reactions, such as swelling or fluid accumulation, were observed in the peripheral bone tissues (Figure 1E and F). Over time, both implant materials and bone tissues became closely connected. The interface between bone and implant materials showed no abnormal reaction. Neither type of implant biomaterials showed any bleaching. At postoperative 24 weeks, only n-CDHA/PAA in the experimental group was covered by a thin layer of newly formed bone tissue (Figure $1 \mathrm{G}$ and $\mathrm{H}$ ).

\section{HE staining of intramuscular embedment of biomaterials}

Morphological analysis of HE staining showed that at postoperative 2 weeks, in both the experimental and the control groups, the implant biomaterials were closely wrapped with newly formed fibrous tissue. No significant fibrous capsule developed around the implant biomaterials. Morphology of the peripheral muscles was normal with no necrosis or dissolution in the muscles. A small number of inflammatory cells (ie, lymphocytes and neutrophils) were found around the implant materials. Edema and neovascularization were observed in the peripheral muscles. At postoperative 4 weeks, the inflammation was significantly relieved with nearly no edema and slightly thinner fibrous tissue surrounding the biomaterials (Figure 2A and B). At postoperative 12 weeks, the inflammation had disappeared and continuous thinning of the fibrous tissue had taken place. Peripheral muscles of the implant materials were arranged normally. No abnormal bleaching was found on the implant materials (Figure 2C and D). Similar results were observed at postoperative 24 weeks (Figure $2 \mathrm{E}$ and F).

\section{X-ray scanning of bone after implantation}

$\mathrm{X}$-ray scanning showed that, at postoperative 2 weeks, the peripheral bone tissues surrounding the n-CDHA/PAA and PE had no significant osteolysis, absorption, necrosis, or osteomyelitis. Both implant biomaterials were closely connected to the bone tissue (Figure 3A). At postoperative 4 weeks, no significant difference in bone density was observed between the bone at the interface of drilled hole and biomaterials or the surrounding bone tissue. Moreover, no bone osteolysis or necrosis was observed (Figure 3B). Over time, both n-CDHA/PAA and PE were closely connected with the bone tissues, which underwent no osteolysis or osteomyelitis. The density of the implanted biomaterial bone at the interfaces of $n-C D H A / P A A$ and PE was 

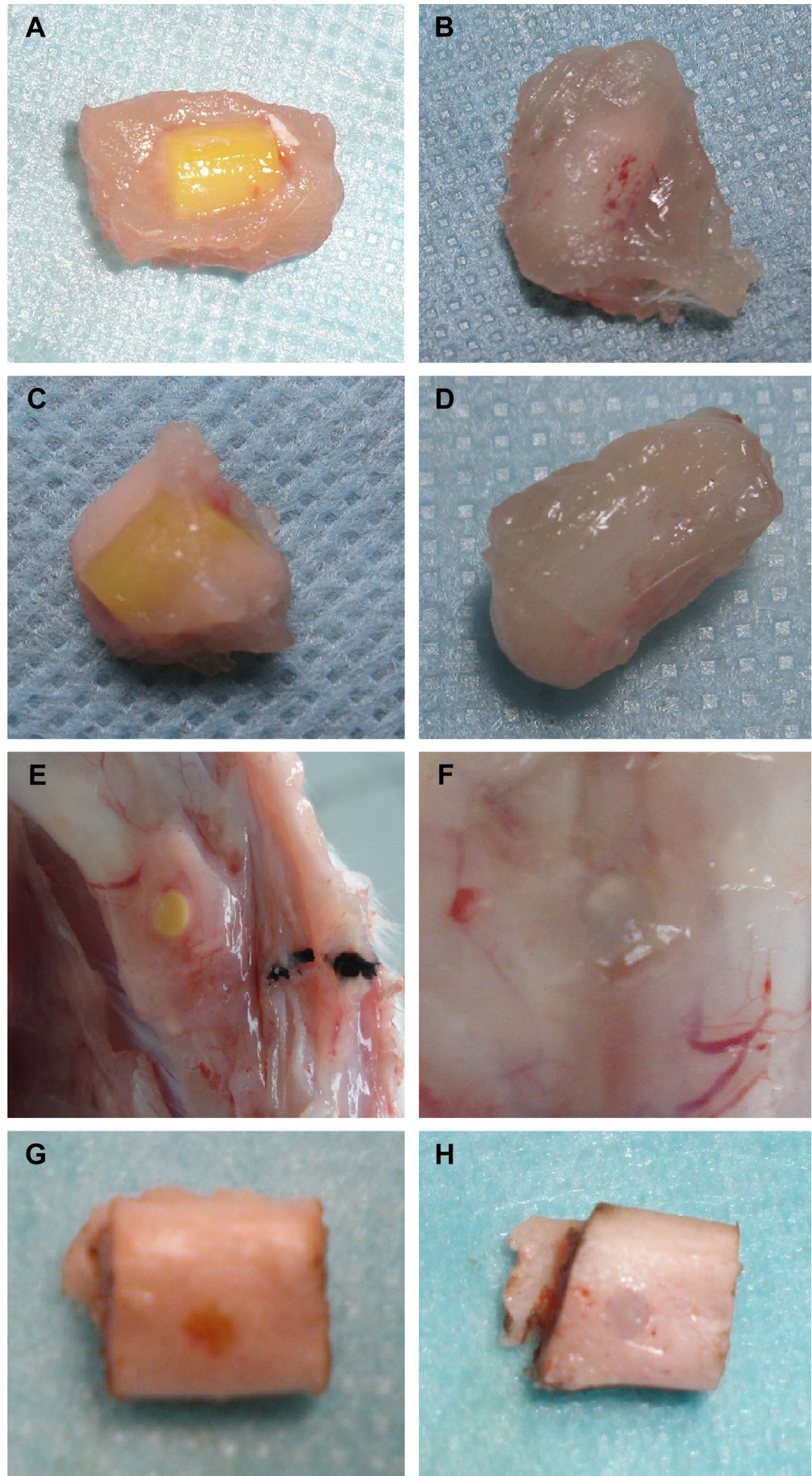

Figure I The results of the muscle and bone implants observed by naked eye in the different groups.

Notes: The specimen of muscle implant operation at 4 weeks in the experimental group (A) and control group (B); 24 weeks in the experimental group (C) and control group (D). The specimen of bone implant operation at 4 weeks in the experimental group $(\mathbf{E})$ and control group $(\mathbf{F}) ; 24$ weeks in the experimental group $(\mathbf{G})$ and control group $(\mathbf{H})$. 

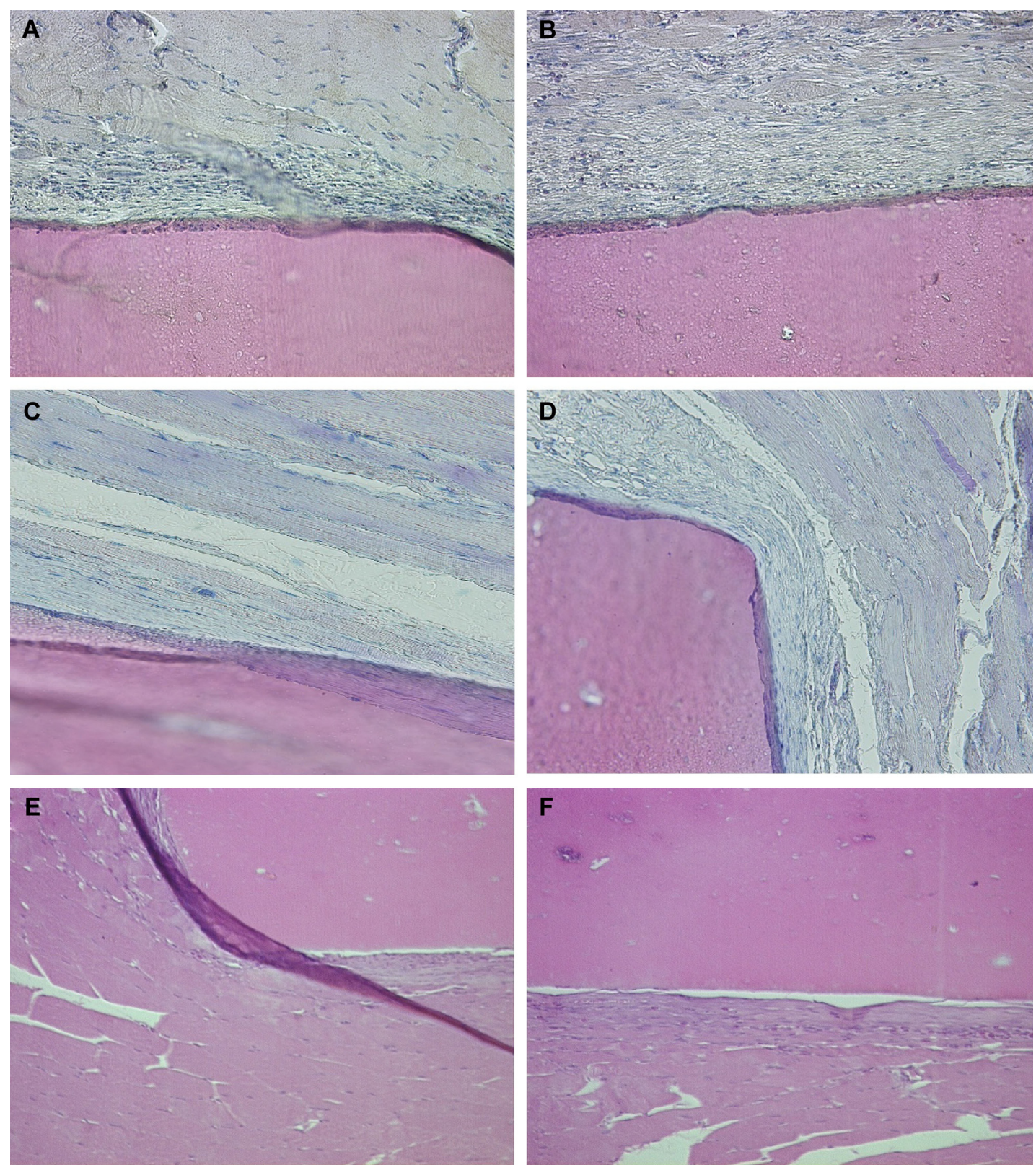

Figure 2 The HE staining of muscle implant operation.

Notes: The HE staining of muscle implant operation at 4 weeks in the experimental group $(\mathbf{A})$ and control group $(\mathbf{B})(\times 200)$; 12 weeks in the experimental group $(\mathbf{C})$ and control group (D) $(\times 200)$; 24 weeks in the experimental group $(\mathbf{E})$ and control group $(\mathbf{F})(\times 200)$.

Abbreviation: $\mathrm{HE}$, hematoxylin and eosin.

consistent with the bone density of the peripheral tissues (Figure 3C and D).

\section{SEM results of bone after biomaterial implantation}

At postoperative 2 weeks, SEM showed that fibrous tissues connected between n-CDHA/PAA and the peripheral bone tissues were formed with deposition of bone mineral matter (Figure 4A). In the control group, only fibrous connective tissues were found between PE and the peripheral bone tissues
(Figure 4B). At postoperative 4 weeks, a large amount of collagen fiber tissue that closely connected the n-CDHA/PAA and the peripheral bone tissues was observed. The results of the control group were similar to those of the 2 weeks. (Figure 4C and D). Bony connections between n-CDHA/ PAA and peripheral bone tissue were observed, and they developed fuzzy boundaries over time. Until postoperative 24 weeks, fibrous tissues between PE and the peripheral bone tissue were closely connected, and small amounts of bone tissue were observed across the PE-bone interface; 

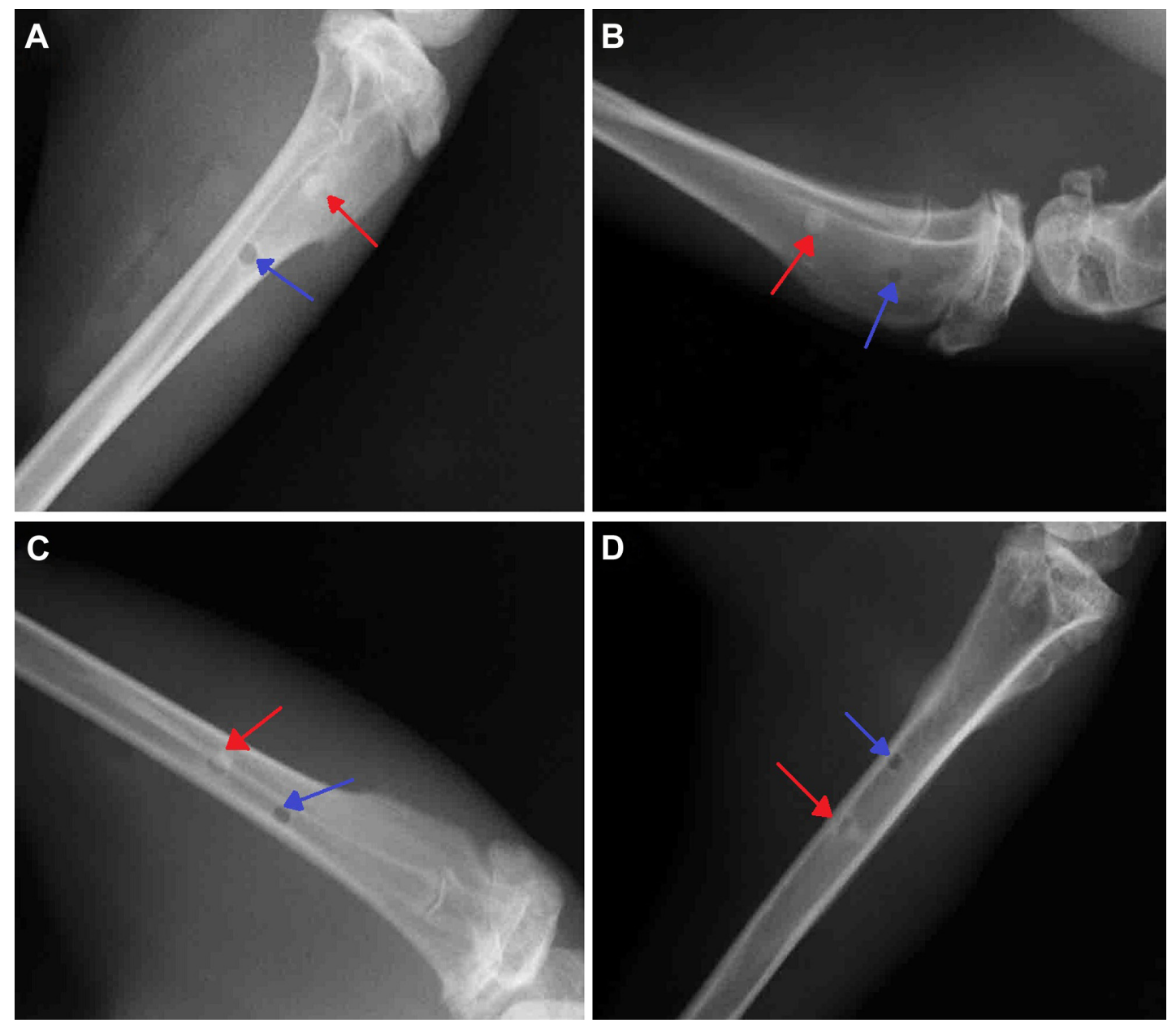

Figure 3 The results of $X$-ray.

Notes: Results of X-ray at 2 weeks (A), 4 weeks (B), 12 weeks (C), and 24 weeks (D) postoperative. The red arrow represents experimental group. The blue arrow represents control group.

however, the surface of n-CDHA/PAA composites were covered with newly formed bone tissue, and no obvious boundaries were observed between n-CDHA/PAA and bone tissue (Figure 4E-H).

\section{Hard-tissue morphology after biomaterial implantation in the bone}

Figures 5-8 show the results of HE, Masson, and toluidine blue staining on hard tissues at different points in time in both groups.

At postoperative 2 weeks (Figure 5), no significant abnormality was observed in the morphology of peripheral bone tissue in the experimental group. The trabecular bone was clear and arranged neatly. Implanted n-CDHA/PAA biomaterials were wrapped in newly generated fibrous tissue with mild invasion of neutrophils and lymphocytes and presence of a small amount of osteoid. The control group was similar to the experimental group.
At postoperative 4 weeks (Figure 6), morphology of peripheral bone tissue in the experimental group was normal, inflammation had subsided, the layer of collagen fibrous tissue had thinned, deposition of newly generated osteoid in the junction between biomaterials and bone tissue had increased, and a small amount of osteogenesis was observed. The control group showed similar results but with less osteoid formation.

At postoperative 12 weeks (Figure 7), the morphologies of trabecular in the peripheral bone of the two groups were good. Implanted n-CDHA/PAA biomaterials were still tightly wrapped in a thin layer of fibrous tissue, and significant increases were observed in the amounts of peripheral bone matrix and osteoid. Osteoid became newly formed trabecular structures, showing the formation of new blood vessels and bone marrow. The neoformative trabecular structures were formed in the control group but were less than the experimental group. 

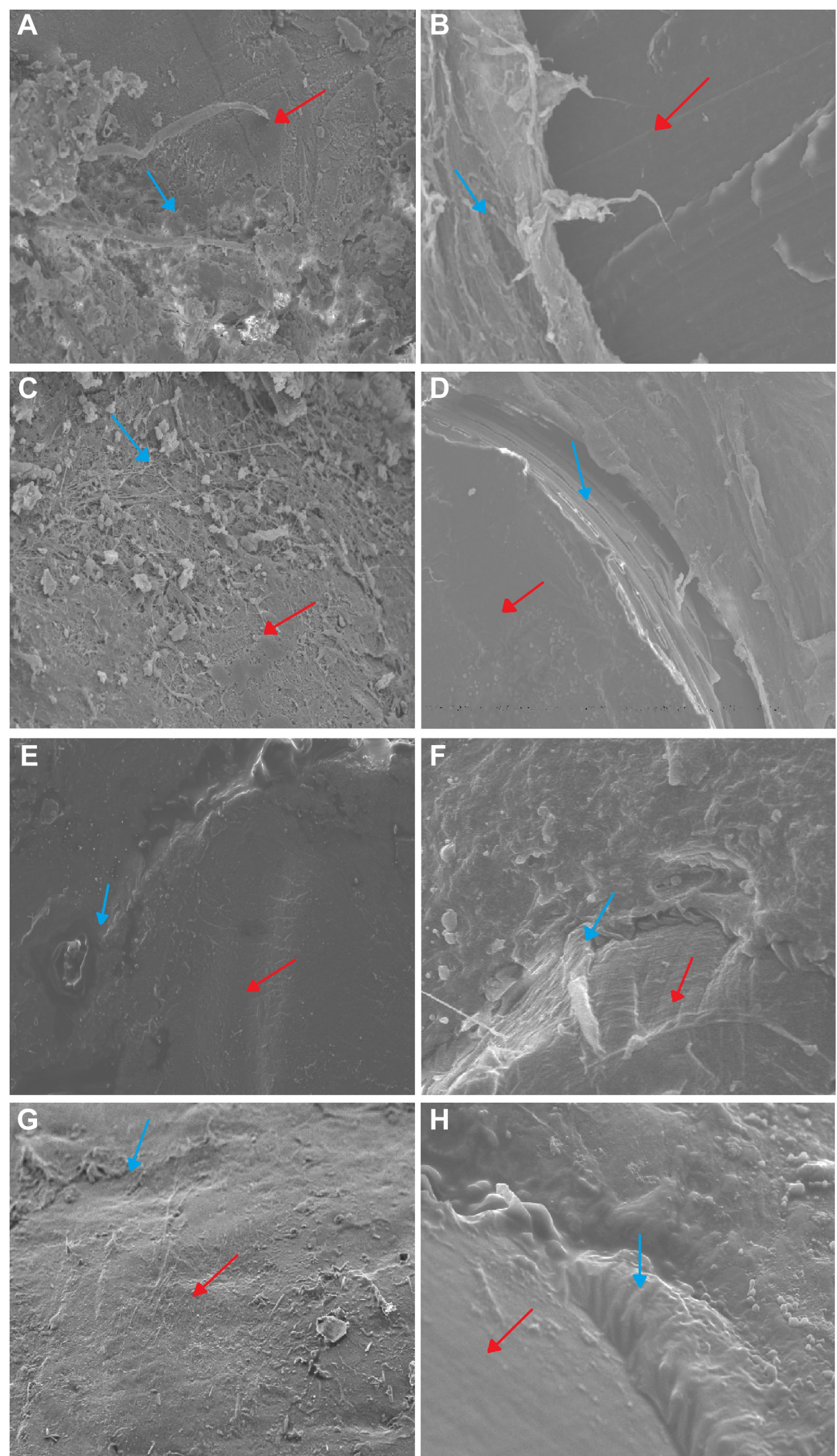

Figure 4 The results of SEM.

Notes: The results of SEM at 2 weeks postoperative in the experimental group $(\mathbf{A}, \times 90)$ and control group $(\mathbf{B}, \times 180) ; 4$ weeks postoperative in the experimental group $(\mathbf{C}, \times 200)$ and control group $(\mathbf{D}, \times 80)$; 12 weeks postoperative in the experimental group $(\mathbf{E}, \times 50)$ and control group $(\mathbf{F}, \times 300)$; 24 weeks postoperative in the experimental group $(\mathbf{G}, \times 350)$ and control group $(\mathbf{H}, \times 35)$. The red arrow represents biomaterials. The blue arrow represents the interface between biomaterial and bone. Abbreviation: SEM, scanning electron microscopy. 

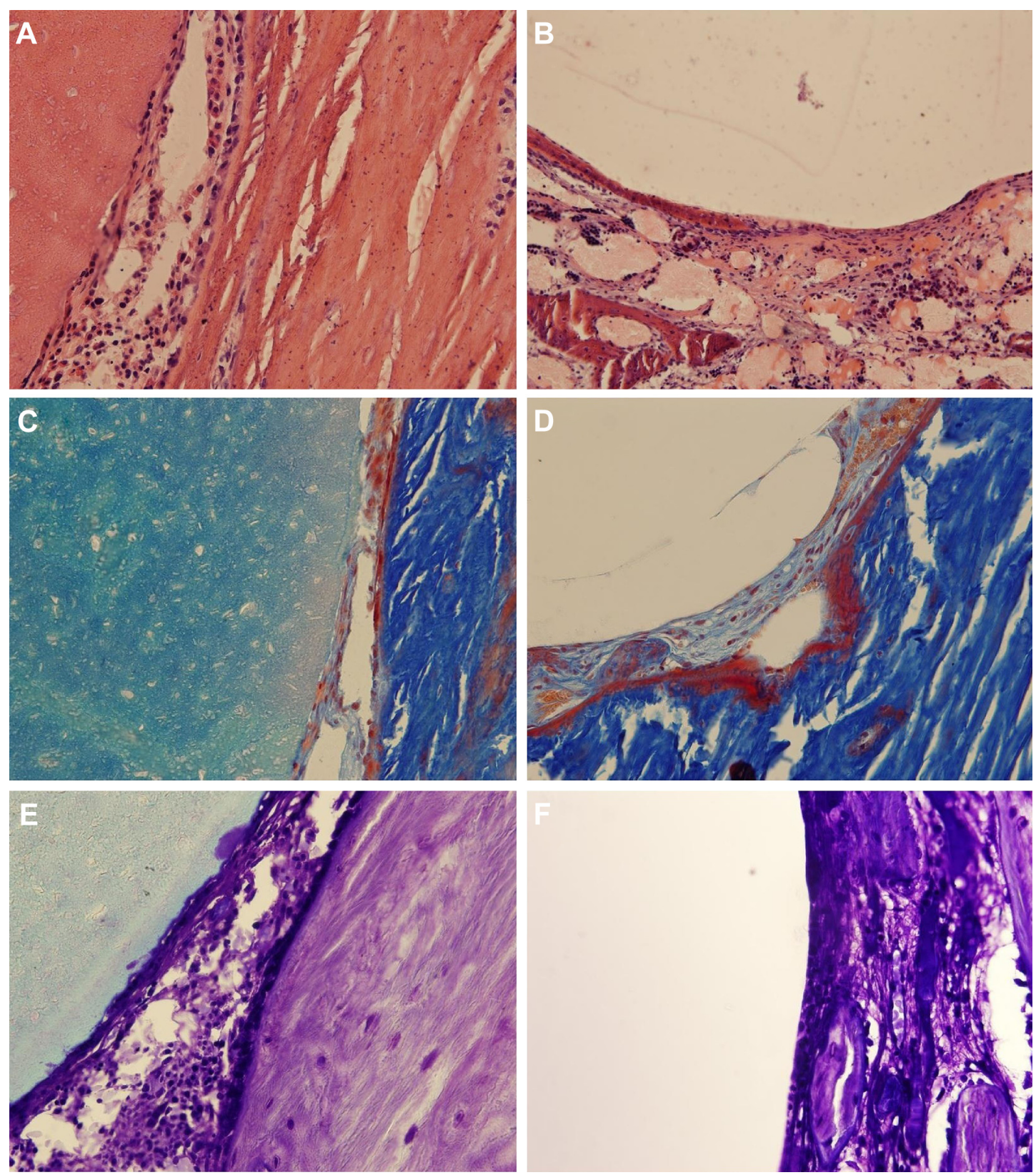

Figure $\mathbf{5}$ The HE, Masson, and toluidine blue staining.

Notes: $(\mathbf{A}, \mathbf{C}, \mathbf{E})$ in the experimental group and $(\mathbf{B}, \mathbf{D}, \mathbf{F})$ control group at 2 weeks postoperative $(\times 200)$.

Abbreviation: HE, hematoxylin and eosin.

At postoperative 24 weeks (Figure 8), no abnormal damage or osteolysis was observed in the peripheral bone tissues of both groups of animals. Peripheral bone tissue of implanted PE biomaterials was closely connected by a layer of fibrous tissue; however, the peripheral bone tissue of implant n-CDHA/PAA biomaterials gradually formed lamellar bones, which were arranged vertically.

\section{Routine blood and biochemical markers}

Tables 1 and 2 show the results of routine blood and serum biochemical analyses of the n-CDHA/PAA group at different points in time. No significant differences were observed in different indices at different points in time before and after the surgery $(P>0.05)$. The new biomaterial implants had no significant effects on routine blood or biochemical markers as indicated by examination of animals after surgery.

\section{Discussion}

In vivo implantation of a biomaterial provides macro and micro levels of evaluation of local and systemic reactions of the biomaterials. Physicochemical properties of the 

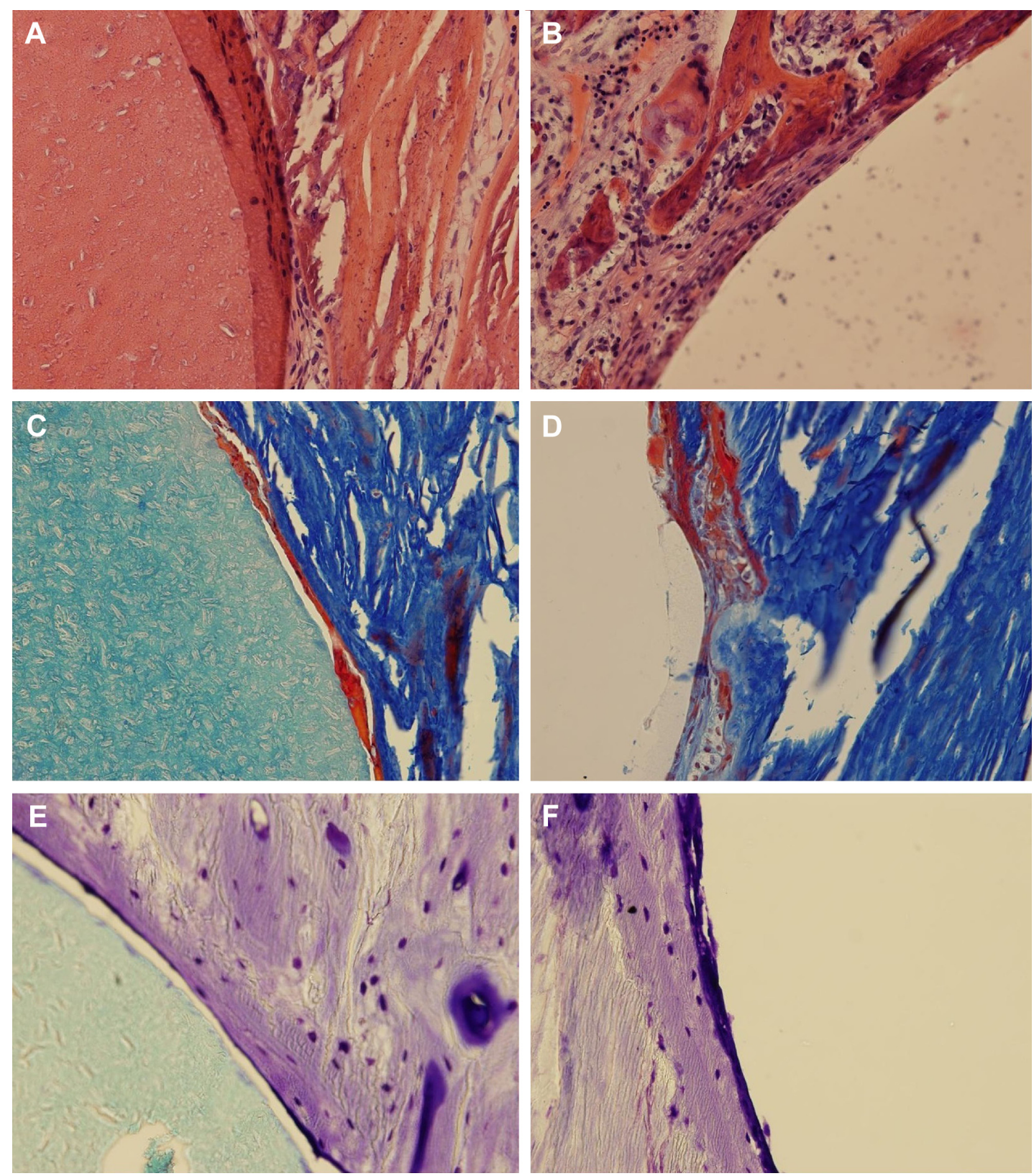

Figure 6 The HE, Masson, and toluidine blue staining.

Notes: $(\mathbf{A}, \mathbf{C}, \mathbf{E})$ in the experimental group and $(\mathbf{B}, \mathbf{D}, \mathbf{F})$ control group at 4 weeks postoperative $(\times 200)$.

Abbreviation: $\mathrm{HE}$, hematoxylin and eosin.

material itself, site or in vivo loading of the implant, and the movement of the implant materials may affect the local tissue or the entire body of the receiver. ${ }^{9,10} \mathrm{PE}$ is a nontoxic biomaterial. It has been used in daily life and clinical medicine for decades and was approved by the Food and Drug Administration in the USA in 1992. It has also been recommended as a control implant biomaterial in in vivo studies by the International Organization of Standardization (ISO). Biomaterials of different categories should be tested with different experimental cycles and implantation sites. This study was performed with reference to published guidelines (ISO10993-1) for the biological evaluation of medical devices and biomaterials from ISO. This information was used to design experiments on intramuscular and bone implantations, which lasted 24 weeks. They were used to assess the histocompatibility of new n-CDHA/PAA composites in in vivo tissues.

Intramuscular implantation of biomaterials facilitates macro and micro levels of evaluation of local reactions in muscle tissue, including assessment of the possibility of inflammation, foreign object rejection, immune response, fibrosis, and other reactions. ${ }^{11,12}$ Visual evaluation and 

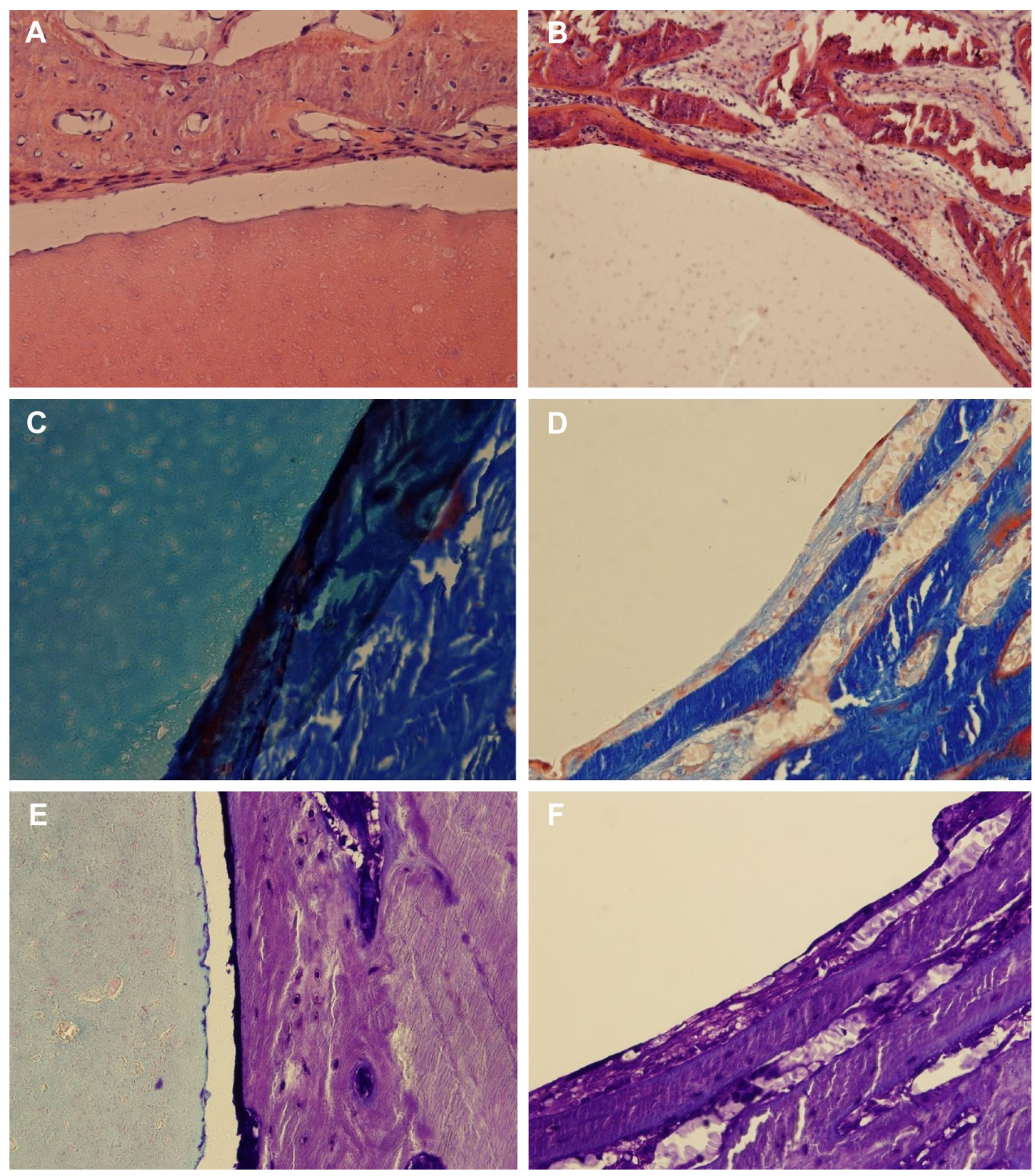

Figure 7 The HE, Masson, and toluidine blue staining.

Notes: $(\mathbf{A}, \mathbf{C}, \mathbf{E})$ in the experimental group and $(\mathbf{B}, \mathbf{D}, \mathbf{F})$ control group at 12 weeks postoperative $(\times 200)$.

Abbreviation: $\mathrm{HE}$, hematoxylin and eosin.

HE staining were carried out and compared between intramuscular implantation of PE and n-CDHA/PAA. No significant necrosis, abnormal inflammation, immune responses, or heterotopic ossification was observed in the peripheral muscle tissue at 24 weeks after implantation. Acute nonspecific inflammation was induced by a small amount of lymphocytes and neutrophils at 2 weeks after implantation. This was presumably caused by surgical trauma, secondary microbial invasion, or implantation-induced local immune response. ${ }^{13,14}$ At 4 weeks after the implantation, this acute inflammation had disappeared from both the experimental and control groups. No inflammation was observed at 12 weeks after the intramuscular implantation of both biomaterials. Implanted biomaterials required only a small amount of fibrous tissue to connect closely to muscle tissues. Implantation of biomaterials in the bone had a very complicated effect on the receivers. In general, four major reactions took place after implantation: 1) necrosis in the peripheral bone tissues, indicating the toxicity of the implanted biomaterials; 2) absorption of implanted biomaterials by the peripheral tissues, causing 

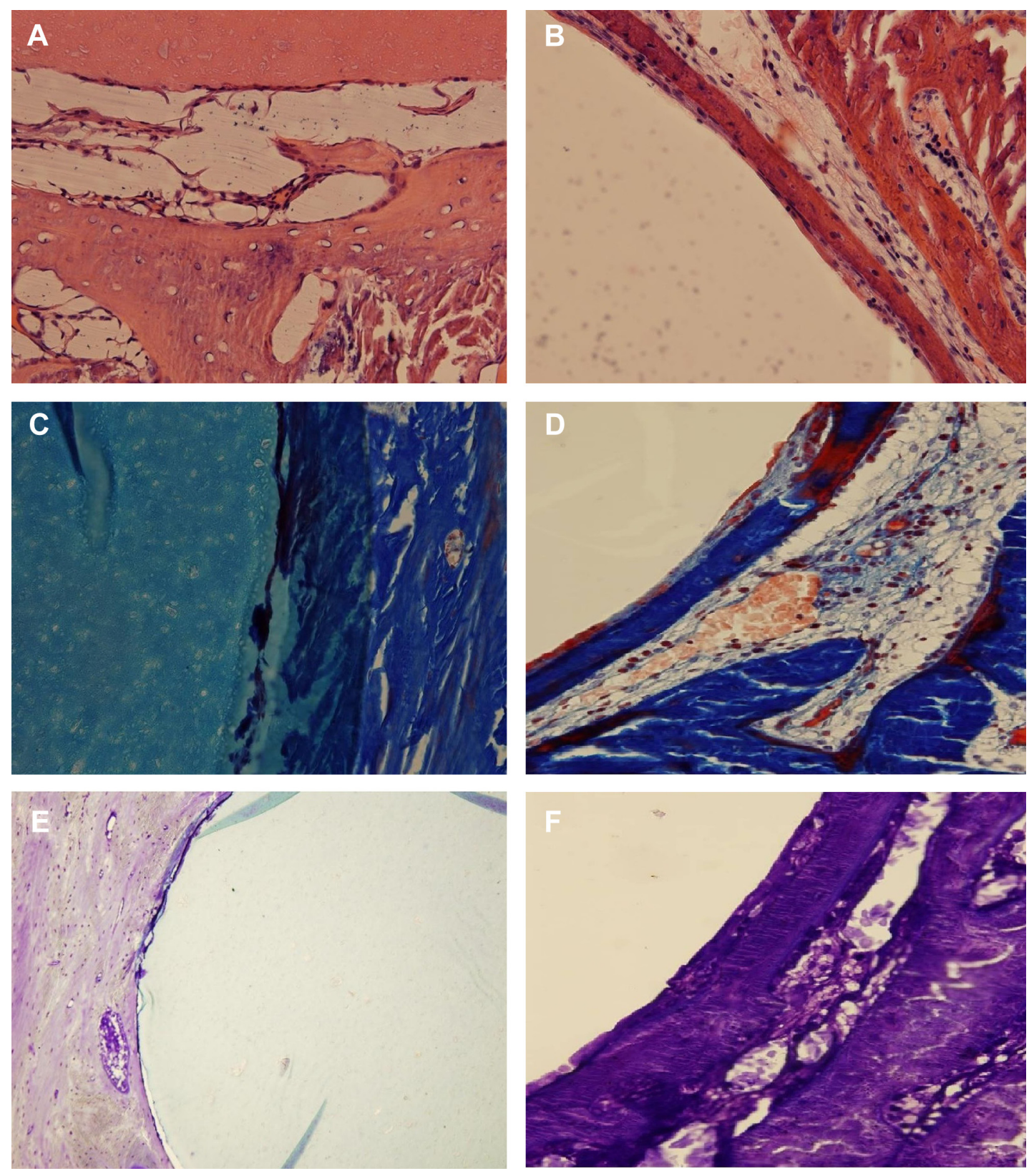

Figure 8 The $\mathrm{HE}$, Masson, and toluidine blue staining.

Notes: $(\mathbf{A}, \mathbf{C}, \mathbf{E})$ in the experimental group and (B, D, F) control group at 24 weeks postoperative $(\times 200)$.

Abbreviation: $\mathrm{HE}$, hematoxylin and eosin.

their disappearance without any degeneration or necrosis, indicating that the implant biomaterials were nontoxic and could be degraded; 3 ) formation of woven fibrous capsule of different thicknesses around the implant biomaterials, suggesting that the biomaterials were biologically inert; and 4) organic combination of implanted biomaterials with the peripheral tissues, indicating that the biomaterials were nontoxic and biologically active. ${ }^{15,16}$ According to the results collected here, the incision showed no significant infection or sinus formation; instead it was healing well after the implantation of n-CDHA/PAA composites in the bones of the animals. Hard-tissue morphology showed no specific pathological changes, such as necrosis and osteomyelitis in the peripheral bone tissues at different points in time after the implantation of both biomaterials. In addition, the eventual outcomes of the peripheral inflammation of both biomaterials were similar, suggesting that n-CDHA/PAA composites and PE had similar effects on the peripheral tissues. Neither caused acute or specific pathological changes. We found that the implanted PE biomaterials were always wrapped in 
Table I The results of blood routine in the $\mathrm{n}-\mathrm{CDHA} / \mathrm{PAA}$ group at different time points $(\bar{x} \pm s, \mathrm{n}=3)$

\begin{tabular}{|c|c|c|c|}
\hline Examination item & Preoperative & 2 weeks & 24 weeks \\
\hline WBC $\left(\times 10^{9} / \mathrm{L}\right)$ & $4.85 \pm 0.95$ & $4.75 \pm 1.035^{\mathrm{a}}$ & $4.82 \pm 0.85^{b}$ \\
\hline Lymphocyte (\%) & $52.25 \pm 8.32$ & $53.02 \pm 10.05^{\mathrm{a}}$ & $51.00 \pm 12.36^{\mathrm{b}}$ \\
\hline Monocyte (\%) & $5.68 \pm 1.85$ & $5.88 \pm 1.58^{\mathrm{a}}$ & $6.04 \pm 1.66^{\mathrm{b}}$ \\
\hline Neutropenia (\%) & $42.56 \pm 11.35$ & $46.53 \pm 13.25^{a}$ & $40.78 \pm 11.23^{b}$ \\
\hline Eosinophil (\%) & $0.38 \pm 0.25$ & $0.42 \pm 0.22^{\mathrm{a}}$ & $0.42 \pm 0.32^{\mathrm{b}}$ \\
\hline Basophil (\%) & $2.55 \pm 1.00$ & $2.62 \pm 0.84^{\mathrm{a}}$ & $2.60 \pm 0.75^{b}$ \\
\hline $\operatorname{RBC}\left(\times 10^{12} / \mathrm{L}\right)$ & $6.32 \pm 0.52$ & $5.88 \pm 0.34^{\mathrm{a}}$ & $6.10 \pm 0.53^{b}$ \\
\hline $\operatorname{HGB}(g / L)$ & $112.20 \pm 7.68$ & || $4.3 \mid \pm 4.25^{\mathrm{a}}$ & $\mathrm{I} 10.45 \pm 5.32^{\mathrm{b}}$ \\
\hline $\mathrm{HCT}$ & $50.62 \pm 6.32$ & $52.4 I \pm 5.33^{\mathrm{a}}$ & $50.12 \pm 4.98^{b}$ \\
\hline MCV (fl) & $82.25 \pm 4.52$ & $77.80 \pm 6.32^{\mathrm{a}}$ & $85.40 \pm 5.55^{b}$ \\
\hline $\mathrm{MCH}(\mathrm{pg})$ & $20.15 \pm 0.47$ & $21.65 \pm 0.54^{\mathrm{a}}$ & $20.47 \pm 0.46^{b}$ \\
\hline $\mathrm{MCHC}(\mathrm{g} / \mathrm{L})$ & $220.35 \pm 6.58$ & $219.87 \pm 4.25^{a}$ & $230.47 \pm 7.46^{b}$ \\
\hline RDW (\%) & $14.85 \pm 2.35$ & $13.74 \pm 3.65^{\mathrm{a}}$ & $15.26 \pm 1.58^{\mathrm{b}}$ \\
\hline $\operatorname{PLT}\left(\times 10^{9} / \mathrm{L}\right)$ & $254.36 \pm 35.246$ & $269.24 \pm 26.59 /^{a}$ & $259.54 \pm 30.684^{b}$ \\
\hline MPV (fl) & $6.88 \pm 0.25$ & $7.03 \pm 0.33^{\mathrm{a}}$ & $6.80 \pm 0.36^{\mathrm{b}}$ \\
\hline РCT & $0.16 \pm 0.02$ & $0.18 \pm 0.04^{\mathrm{a}}$ & $0.16 \pm 0.05^{b}$ \\
\hline
\end{tabular}

Notes: ${ }^{a} P>0.05$, compared with the preoperative; ${ }^{b} P>0.05$, compared with the preoperative.

Abbreviations: n-CDHA/PAA, nano-calcium-deficient hydroxyapatite/poly-amino acid; WBC, white blood cell; RBC, red blood cell; HGB, hemoglobin; HCT, hematocrit; MCV, mean corpuscular volume; MCH, mean corpuscular hemoglobin; MCHC, mean corpuscular hemoglobin concentration; RDW, red cell distribution width; PLT, platelet; MPV, mean platelet volume; PCT, procalcitonin.

fibrous connective tissue. In contrast, the areas surrounding the n-CDHA/PAA composites gradually showed increased bone mass, newly formed trabecular bone, neovasculature, and formation of lamellar bone. These results indicated that n-CDHA/PAA composites were nontoxic and bioactive.

Interaction between foreign biomaterials and bone tissues, specifically the healing mechanism of material-bone interface, had a profound effect on the functions of biomaterial implants and on prognosis. In general, after the in vivo implantation of biomaterials into the bone, biomaterials had direct contact with a variety of matrix components and proteins of body tissue fluid and blood. They also adsorbed the surrounding biological macromolecules from tissue and blood, such as bone laminin, fibronectin, fibrinogen, various bone morphogenetic proteins, and other cytokines, to form a layer of biological macromolecules, thereby causing a

Table 2 The results of biochemical indicator in the $n-C D H A / P A A$ group at different time points $(\bar{x} \pm s, n=3)$

\begin{tabular}{llll}
\hline Examination item & Preoperative & $\mathbf{2}$ weeks & $\mathbf{2 4}$ weeks \\
\hline ALT $(\mathrm{U} / \mathrm{L})$ & $2.36 \pm 0.56$ & $2.61 \pm 0.23^{\mathrm{a}}$ & $2.71 \pm 0.5 \mathrm{I}^{\mathrm{b}}$ \\
AST $(\mathrm{U} / \mathrm{L})$ & $1.44 \pm 0.35$ & $1.35 \pm 0.28^{\mathrm{a}}$ & $1.39 \pm 0.45^{\mathrm{b}}$ \\
TP $(\mathrm{g} / \mathrm{L})$ & $67.88 \pm 6.52$ & $65.28 \pm 5.7^{\mathrm{a}}$ & $66.59 \pm 7.24^{\mathrm{b}}$ \\
Alb $(\mathrm{g} / \mathrm{L})$ & $53.59 \pm 4.68$ & $56.32 \pm 3.4^{\mathrm{a}}$ & $54.68 \pm 4.21^{\mathrm{b}}$ \\
TBIL $(\mu \mathrm{mol} / \mathrm{L})$ & $4.61 \pm 0.55$ & $4.92 \pm 0.4^{\mathrm{a}}$ & $4.90 \pm 0.44^{\mathrm{b}}$ \\
Glu $(\mathrm{mmol} / \mathrm{L})$ & $7.56 \pm 1.23$ & $7.85 \pm 0.58^{\mathrm{a}}$ & $7.45 \pm 0.86^{\mathrm{b}}$ \\
BUN $(\mathrm{mmol} / \mathrm{L})$ & $10.23 \pm 1.58$ & $11.35 \pm 1.23^{\mathrm{a}}$ & $11.32 \pm 0.99^{\mathrm{b}}$ \\
CREA $(\mu \mathrm{mol} / \mathrm{L})$ & $156.25 \pm 16.35$ & $163.49 \pm 15.42^{\mathrm{a}}$ & $160.87 \pm 18.42^{\mathrm{b}}$ \\
UA $(\mu \mathrm{mol} / \mathrm{L})$ & $7.23 \pm 2.36$ & $7.56 \pm 4.23^{\mathrm{a}}$ & $7.44 \pm 2.14^{\mathrm{b}}$ \\
TC $(\mu \mathrm{mol} / \mathrm{L})$ & $1.35 \pm 0.33$ & $1.34 \pm 0.26^{\mathrm{a}}$ & $1.30 \pm 0.16^{\mathrm{b}}$ \\
TG $(\mu \mathrm{mol} / \mathrm{L})$ & $0.77 \pm 0.32$ & $0.80 \pm 0.23^{\mathrm{a}}$ & $0.82 \pm 0.19^{\mathrm{b}}$ \\
HDL $(\mu \mathrm{mol} / \mathrm{L})$ & $23.65 \pm 5.42$ & $22.69 \pm 3.48^{\mathrm{a}}$ & $22.86 \pm 4.26^{\mathrm{b}}$ \\
LDL $(\mu \mathrm{mol} / \mathrm{L})$ & $12.30 \pm 4.68$ & $13.00 \pm 3.56^{\mathrm{a}}$ & $12.82 \pm 4.36^{\mathrm{b}}$ \\
LDH $(\mathrm{U} / \mathrm{L})$ & $5.55 \pm 2.32$ & $5.34 \pm 2.64^{\mathrm{a}}$ & $5.32 \pm 2.14^{\mathrm{b}}$ \\
A/G & $6.66 \pm 2.15$ & $6.92 \pm 2.36^{\mathrm{a}}$ & $6.89 \pm 2.85^{\mathrm{b}}$ \\
GGT $(\mathrm{U} / \mathrm{L})$ & $86.49 \pm 26.48$ & $87.64 \pm 24.69^{\mathrm{a}}$ & $88.64 \pm 26.14^{\mathrm{b}}$ \\
ALP $(\mathrm{U} / \mathrm{L})$ & $0.69 \pm 0.46$ & $0.85 \pm 0.36^{\mathrm{a}}$ & $0.79 \pm 0.42^{\mathrm{b}}$ \\
\hline
\end{tabular}

Notes: ${ }^{a} P>0.05$, compared with the preoperative; ${ }^{b}>0.05$, compared with the preoperative.

Abbreviations: n-CDHA/PAA, nano-calcium-deficient hydroxyapatite/poly-amino acid; ALT, alanine transaminase; AST, aspartate aminotransferase; TP, total protein; Alb, albumin; TBIL, increased total bilirubin; Glu, glucose; BUN, blood urea nitrogen; CREA, creatinine; UA, uric acid; TC, total count; TG, triglycerides; HDL, high-density lipoprotein; LDL, Low-density lipoprotein; LDH, lactate dehydrogenase; A/G, albumin/globulin; GGT, gamma-glutamyl transpeptidase; ALP, alkaline phosphatase. 
series of cytological changes. Cellular transformation factors induce the undifferentiated osteoblasts, mesenchymal cells, and osteoblasts to migrate and adhere to the surface of the biomaterials through cellular adhesion. Undifferentiated mesenchymal cells begin to proliferate and differentiate through the reactions of growth factors in the cells. ${ }^{17-19}$ The initial properties of the surfaces of the biomaterials determined the type and the quantity of protein adsorption, thereby affecting the binding status of the host cells and the surface of the materials. ${ }^{20,21}$ Compared with the n-HA, n-CDHA has higher solubility (ie, degradability) at a lower $\mathrm{Ca} / \mathrm{P}$ ratio (1.5-1.67), as well as being more similar in composition and crystal structure to the mineral of natural bone..$^{22,23}$ Thus, it is envisaged that n-CDHA can be fabricated as a novel bone regeneration material in order to get better bioperformance of apatite biomaterial. ${ }^{24,25}$ The PAA copolymer could be degraded in HCL-Tris solution with weight loss of 30 wt $\%$ after 12 weeks of soaking, and it had no significant effect on the $\mathrm{pH}$ value of the ambient environment during the degradation period. ${ }^{26}$ If the $\mathrm{pH}$ value in the ambient solution was decreased, it was believed to induce the inflammatory reaction in vivo. ${ }^{27}$ Cell culture experiments and in vivo implantation results showed that the PAA also had good biocompatibility. ${ }^{26}$ When implanted in cortical bone of the dogs, the PAA copolymer implants were directly connected with the host bone tissue without obvious intervening connective layer, and some new bone tissues were found to extend along the copolymer surface, which was known as bone-bonding. ${ }^{26}$ Bone-bonding could ensure that the implant integrated with natural bone through biochemical reaction at the interface between biomaterials and bone tissue, which was in favor of implant fixation in host bone. ${ }^{28}$ Therefore, these previous experiments suggested that the combination of PAA with n-CDHA would not have apparent adverse reaction with the bone tissue and surrounding biological macromolecules from tissue and blood, and it may have excellent biocompatibility and osteoconductivity as potential implants in orthopedic surgery. Histological and X-ray scanning results in this study indicated that the majority of the biomaterials could connect closely to the peripheral bone tissues 2 weeks after the implantation into the bone. Light microscopy and Masson and toluidine blue stained tissues showed osteoid deposition on the material-bone interface 4 weeks after the implantation. The amount of bone tissue formed increased over time. Mineralization and reconstruction increased the strength of the new bone, and the trabecular structure had formed by 12 weeks after implantation. Results of SEM showed that new bone tissue had crossed the material-bone interface and had grown on the surface of the biomaterials by 12 weeks after the implantation. By 24 weeks, the implant biomaterials had become completely covered by new bone tissues. The results of histological staining showed that the n-CDHA/ PAA composites did not have a stationary relationship but rather a strong biological bond with the bone-tissue interface. Further analyses of the specific mechanisms underlying this bond will be necessary in further studies.

\section{Conclusion}

The compatibility of n-CDHA/PAA biocomposite was evaluated with muscle and bone tissue in an in vivo model. The results showed that no manifest inflammation was observed after the implantation of n-CDHA/PAA in vivo and a strong biological bond formed between implant and the bone tissue, indicating that the composites were safe, nontoxic, and biologically active in bone repair.

\section{Acknowledgments}

This work was supported by international S\&T cooperation "Cooperative research on advanced composite biological materials and clinical products" Program (Grant No 2013DFB50280) and Key Technologies R\&D Program of Sichuan province (Grant No 2014GZX0010).

\section{Disclosure}

The authors report no conflicts of interest in this work.

\section{References}

1. Aurégan JC, Bégué T. Induced membrane for treatment of critical sized bone defect: a review of experimental and clinical experiences. Int Orthop. 2014;38(9):1971-1978.

2. Kleinschmidt K, Wagner-Ecker M, Bartek B, Holschbach J, Richter W. Superior angiogenic potential of GDF-5 and GDF-5(V453/V456) compared with BMP-2 in a rabbit long-bone defect model. J Bone Joint Surg Am. 2014;96(20):1699-1707.

3. Reichert JC, Saifzadeh S, Wullschleger ME, et al. The challenge of establishing preclinical models for segmental bone defect research. Biomaterials. 2009;30(12):2149-2163.

4. Hesse E, Kluge G, Atfi A, et al. Repair of a segmental long bone defect in human by implantation of a novel multiple disc graft. Bone. 2010;46(5): 1457-1463.

5. Li H, Gong M, Yang A, Ma J, Li X, Yan Y. Degradable biocomposite of nano calcium-deficient hydroxyapatite-multi(amino acid) copolymer. Int J Nanomedicine. 2012;7:1287-1295.

6. Li H, Yang L, Dong X, Gu Y, Lv G, Yan Y. Composite scaffolds of nano calcium deficient hydroxyapatite/multi-(amino acid)copolymer for bone tissue regeneration. J Mater Sci Mater Med. 2014;25(5):1257-1265.

7. Bogdanović U, Vodnik V, Mitrić M, et al. Nanomaterial with high antimicrobial efficacy-copper/polyaniline nanocomposite. ACS Appl Mater Interfaces. 2015;7(3):1955-1966.

8. Yao Q, Ye J, Xu Q, Mo A, Gong P. Composite scaffolds of dicalcium phosphate anhydrate/multi-(amino acid) copolymer: in vitro degradability and osteoblast biocompatibility. J Biomater Sci Polym Ed. 2015;26(4): 211-223.

9. Sancey L, Kotb S, Truillet C, et al. Long-term in vivo clearance of gadolinium-based AGuIX nanoparticles and their biocompatibility after systemic injection. ACS Nano. 2015;9(3):2477-2488. 
10. Watari F, Yokoyama A, Omori M, et al. Biocompatibility of materials and development to functionally graded implant for bio-medical application. Compos Sci Technol. 2004;64(6):893-908.

11. Walker J, Shadanbaz S, Woodfield TB, Staiger MP, Dias GJ. The in vitro and in vivo evaluation of the biocompatibility of $\mathrm{Mg}$ alloys. Biomed Mater. 2014;9(1):015006.

12. Wang H, Zhi W, Lu X, et al. Comparative studies on ectopic bone formation in porous hydroxyapatite scaffolds with complementary pore structures. Acta Biomater. 2013;9(9):8413-8421.

13. Kamelger FS, Marksteiner R, Margreiter E, et al. A comparative study of three different biomaterials in the engineering of skeletal muscle using a rat animal model. Biomaterials. 2004;25(9):1649-1655.

14. Srivastava S, Gorham SD, French DA, Shivas AA, Courtney JM. In vivo evaluation and comparison of collagen, acetylated collagen and collagen/glycosaminoglycan composite films and sponges as candidate biomaterials. Biomaterials. 1990;11(3):155-161.

15. Gauthier O, Müller R, von Stechow D, et al. In vivo bone regeneration with injectable calcium phosphate biomaterial: a three-dimensional micro-computed tomographic, biomechanical and SEM study. Biomaterials. 2005;26(27):5444-5453.

16. Chu CL, Xue XY, Zhu JC, Yin ZD. In vivo study on biocompatibility and bonding strength of Ti/Ti-20 vol.\% HA/Ti-40 vol.\% HA functionally graded biomaterial with bone tissues in the rabbit. Mat Sci Eng A Struct. 2006;429(1-2):18-24.

17. Roessler S, Born R, Scharnweber D, Worch H, Sewing A, Dard M. Biomimetic coatings functionalized with adhesion peptides for dental implants. J Mater Sci Mater Med. 2001;12(10-12):871-877.

18. Chehroudi B, Gould TR, Brunette DM. A light and electron microscopic study of the effects of surface topography on the behavior of cells attached to titanium-coated percutaneous implants. J Biomed Mater Res. 1991;25(3):387-405.
19. Kirkpatrick CJ, Wagner M, Köhler H, Bittinger F, Otto M, Klein CL. The cell and molecular biological approach to biomaterial research: a perspective. J Mater Sci Mater Med. 1997;8(3):131-141.

20. Ziats NP, Miller KM, Anderson JM. In vitro and in vivo interactions of cells with biomaterials. Biomaterials. 1988;9(11):5-13.

21. Wang H, Lee JK, Moursi AM, et al. Microstructural disassembly of calcium phosphates. J Biomed Mater Res A. 2004;68(1):61-70.

22. Guo H, Su J, Wei J, Kong H, Liu C. Biocompatibility and osteogenicity of degradable Ca-deficient hydroxyapatite scaffolds from calcium phosphate cement for bone tissue engineering. Acta Biomater. 2009;5(1): 268-278.

23. Lin JH, Kuo KH, Ding SJ, Ju CP. Surface reaction of stoichiometric and calcium-deficient hydroxyapatite in simulated body fluid. J Mater Sci Mater Med. 2001;12(8):731-741.

24. Gustavsson J, Ginebra MP, Planell J, Engel E. Osteoblast-like cellular response to dynamic changes in the ionic extracellular environment produced by calcium-deficient hydroxyapatite. J Mater Sci Mater Med. 2012;23(10):2509-2520.

25. Akiyama N, Takemoto M, Fujibayashi S, Neo M, Hirano M, Nakamura T. Difference between dogs and rats with regard to osteoclast-like cells in calcium-deficient hydroxyapatite-induced osteoinduction. J Biomed Mater Res A. 2011;96(2):402-412.

26. Li H, Yan Y, Wei J, et al. Bone substitute biomedical material of multi(amino acid) copolymer: in vitro degradation and biocompatibility. J Mater Sci Mater Med. 2011;22(11):2555-2563.

27. Ambrose CG, Clanton TO. Bioabsorbable implants: review of clinical experience in orthopedic surgery. Ann Biomed Eng. 2004;32(1): 171-177.

28. Davies JE. Bone bonding at natural and biomaterial surfaces. Biomaterials. 2007;28(34):5058-5067.
International Journal of Nanomedicine

\section{Publish your work in this journal}

The International Journal of Nanomedicine is an international, peerreviewed journal focusing on the application of nanotechnology in diagnostics, therapeutics, and drug delivery systems throughout the biomedical field. This journal is indexed on PubMed Central, MedLine, CAS, SciSearch ${ }^{\circledR}$, Current Contents ${ }^{\circledR} /$ Clinical Medicine,

\section{Dovepress}

Journal Citation Reports/Science Edition, EMBase, Scopus and the Elsevier Bibliographic databases. The manuscript management system is completely online and includes a very quick and fair peer-review system, which is all easy to use. Visit http://www.dovepress.com/ testimonials.php to read real quotes from published authors. 